\title{
Dynamic output feedback sliding mode control for uncertain linear systems
}

\section{Jiehua Feng', Dongya Zhao', Xing-Gang Yan², Sarah K. Spurgeon $^{3}$}

\begin{abstract}
In this paper, a class of uncertain linear systems with unmatched disturbances is considered, where the nominal system representation is allowed to be nonminimum phase. A sliding surface is designed which is dependent on the system output, observed state and estimated uncertain parameters. A linear coordinate transformation is introduced so that the stability analysis of the reduced-order sliding mode dynamics can be conveniently performed. A robust output feedback sliding mode control (OFSMC) is then designed to drive the considered system state to reach the sliding surface in finite time and maintain a sliding motion thereafter. $A$ simulation example for a high incidence research model (HIRM) aircraft is used to demonstrate the effectiveness of the proposed method.
\end{abstract}

\section{Keywords}

Sliding mode control; Non-minimum phase; Dynamical output feedback; Regular form; Unmatched disturbances

\section{Introduction}

Physical systems are unavoidably affected by external disturbances and plant uncertainties which may in turn influence the system performance. Sliding mode control

\footnotetext{
${ }^{1}$ College of New Energy, China University of Petroleum (East China), Qingdao, China

${ }^{2}$ School of Engineering \& Digital Arts, University of Kent, United Kingdom

${ }^{3}$ Department of Electronic \& Electrical Engineering, University College London, United Kingdom

Corresponding author:

Dongya Zhao, College of New Energy, China University of Petroleum (East China), Qingdao, China.

Email:dyzhao@upc.edu.cn
} 
(Jiang et al., (2021b)) has been widely studied as an effective method to tackle this problem and has been employed in some industrial settings due to its conceptual simplicity and strong robustness properties. Much of the early work on sliding mode control assumed that all system states can be measured (Utkin $(1977,1992)$ ). However, in actual engineering systems, this assumption may be unreasonable. This has motivated the current emphasis on the development of output feedback sliding mode control (OFSMC).

Many OFSMC algorithms have been proposed to stabilize uncertain systems. A static output feedback variable structure control has been investigated for linear systems without disturbances in Elkhazali and Decarlo (1995). A further static OFSMC has been designed for uncertain linear systems with matched disturbances in Edwards and Spurgeon $(1995,1998)$, where a convenient coordinate transformation has been given to obtain the regular form. However, unmatched disturbances act on many practical systems. These unmatched disturbances directly affect the dynamics of the system when the state moves on the sliding surface and exhibits a sliding mode. The well-known Constrained Lyapunov Problem (CLP) has been widely discussed in the literature and some effective methods have been proposed in Galimidi and Barmish (1986); Edwards et al., (2007) to constructively solve this problem. This work facilitated the development of static OFSMC schemes which tackle the problem of unmatched disturbances Yan et al., (2009a, 2013). However, the literature above requires that the plant under consideration is minimum phase. In practice, non-minimum phase systems may be encountered, such as flexible manipulators (Benosman and Vey (2003)) and the underactuated ship (Consolini and Tosques (2012)).

The study of OFSMC for non-minimum phase systems has recently received great attention. A dynamic OFSMC with a dynamic compensator has been proposed to stabilize a class of uncertain linear systems in Yan et al., (2004), where the nominal system may be non-minimum phase. The results have also been extended to timedelay systems in Yan et al., (2009b, 2010). Furthermore, based on the method proposed in Yan et al., (2004) and a reduced-order compensator, a class of large-scale interconnected systems has been stabilized in Yan et al., (2006). Although the case of more general unmatched disturbances with nonlinear bounds has been considered in the above literature, the unmatched disturbances are required to have zero steadystate values or bounded $H_{2}$ norms. In addition, some classic control tools, such as the Riccati approach (Kim et al., (2000)), the LMI-based approach (Park et al., (2007)) and an adaptive approach (Wen and Cheng (2008); Jiang et al., (2021a)) have been used to extend the sliding mode control approach to deal with unmatched disturbances. However, these methods also require that the unmatched disturbances must belong to somewhat restrictive classes of vanishing disturbance, which may be unreasonable in practice (Yang et al., (2013)).

By adding integral action to the sliding surface, integral sliding mode control has become well established and regarded as an effective method to counteract unmatched disturbances with nonzero steady-state value (Zhang and Yan (2019); Cao and Xu (2004)). However, the integral action may cause a large overshoot in the controlled system. More recently, based on the nonlinear disturbance observer proposed in Chen 
(2003); Chen et al., (2000), the observed disturbances have been added to the sliding surface to counteract their effects in Yang et al., (2013). This method has attracted a lot of attention due to its simplicity and effectiveness but can only handle a class of secondorder systems and lacks a strict stability proof of the sliding mode dynamics. The result has been extended for a nominal system representation that is a series of integrators in Ginoya et al., (2013), where a modified sliding surface has been presented to guarantee the practical stability of the overall system. An extended state observer-based sliding mode control has been designed for pulse-width modulation-based DC-DC buck converter systems in Wang et al., (2015). A disturbance observer-based continuous finitetime sliding mode control has been designed for input affine nonlinear systems in Nguyen et al., (2020), where a nonlinear sliding surface and supertwisting algorithm have been used to guarantee finite-time convergence of the considered system. In addition, disturbance observer-based control methods have also been designed for some uncertain nonlinear systems with unmatched disturbances (Yang et al., (2012); Zhang et al., (2017)). However, the literature mentioned above all requires that the considered system states are accessible. These solutions are frequently motivated by engineering practice and using a disturbance observer in dynamic OFSMC may be effective for uncertain non-minimum phase systems but it should be noted that the theoretical analysis may be challenging.

When the system states cannot be measured and the considered system is nonminimum phase, disturbance observer-based sliding mode control design will face new challenges which include: how to observe the system states and unmatched disturbances simultaneously and how to prove the stability of the associated extended system when the system is restricted on the designed sliding surface. For these challenges, a disturbance observer-based dynamic OFSMC strategy is proposed for a class of uncertain linear systems, where the nominal system representation is allowed to be non-minimum phase. Inspired by Yang et al., (2013), a novel sliding surface which is a function of an augmented space formed by the system output, the observed state and the estimated uncertain parameters, is designed. Furthermore, a new linear coordinate transformation has been derived so that the stability analysis of the reduced-order sliding mode dynamics can be conveniently performed. In comparison with existing static OFSMC methods (Gao et al., (2019); Ji et al., (2019)), the nominal part of the system considered in this paper is allowed to be non-minimum phase. In comparison with most of the existing dynamics OFSMC methods (Consolini and Tosques (2012); Yan et al., (2004, 2009b, $2010,2006)$ ), the system considered in this paper is allowed to have the unmatched disturbances with nonzero steady-state value. In comparison with most of the existing disturbance observer-based sliding mode control methods (Yang et al., (2013); Nguyen et al., (2020); Ginoya et al., (2013); Wang et al., (2015)), the system state in this paper is not required to be measurable while the unmatched disturbances have more general forms. The main theoretical contributions of this paper include: (i) a novel sliding surface is proposed to counteract the unmatched disturbances when only the system output information is available; (ii) a sufficient condition is given to guarantee the considered system exhibits an exponentially stable sliding motion while the state observed error and parameter estimation error converge to zero exponentially; (iii) a 
new linear coordinate transformation is proposed to facilitate the stability analysis of the sliding motion dynamics.

The remainder of the paper is organized as follows. Section II formulates the problem and gives some assumptions that will be used in the following sections. In Section III, a novel sliding surface and the stability analysis of the sliding motion is given. A sliding mode control is designed in Section IV. A simulation example of a HIRM aircraft is presented to validate the proposed approach in Section $\mathrm{V}$ while the conclusions are given in Section VI.

Notation: For a square matrix $A, \underline{\lambda}(A)$ and $\bar{\lambda}(A)$ denote the minimum and maximum eigenvalue respectively.

\section{System description and problem formulation}

Consider an uncertain linear system

$$
\begin{aligned}
& \dot{\tilde{x}}=\tilde{A} \tilde{x}+\tilde{B} u+\tilde{\Psi}(t) \theta \\
& y=\tilde{C} \tilde{x}
\end{aligned}
$$

where $\tilde{x} \in R^{n}, y \in R^{p}, u \in R^{m}$ are the system state, output and input vectors respectively with $m \leq p<n, \tilde{A}, \tilde{B}, \tilde{C}$ are constant matrices with appropriate dimensions, $\tilde{\Psi} \in R^{n \times q}$ is a known matrix, and $\theta \in R^{q}$ is an unknown constant parameter vector.

Remark 1. It should be noted that although the considered nominal system is linear, most existing sliding mode control methods for nonlinear systems cannot be directly applied to system (1). For instance, a novel second-order sliding mode control has been proposed for a class of nonlinear systems to handle the unmatched disturbances in Ding and Li (2017), but it is difficult to verify the sufficient conditions proposed to guarantee the stability of the sliding mode dynamics. Moreover, some control parameters in the sliding mode control of nonlinear systems may be difficult to obtain (Plestan et al., (2010); Mu et al., (2017)).

The following Assumptions are imposed on system (1).

Assumption 1. System (1) is controllable and observable.

Assumption 2. $\tilde{B}$ and $\tilde{C}$ are both full rank, $\operatorname{rank}(\tilde{C} \tilde{B})=m$.

Under Assumption 2, it follows from Lemma 5.3 in Edwards and Spurgeon (1998) that there exists a nonsingular coordinate transformation $x=\tilde{T} \tilde{x}$ such that the system (1) can be described as:

$$
\begin{aligned}
& \dot{x}=A x+B u+\Psi(t) \theta \\
& y=C x
\end{aligned}
$$

where $A=\left[\begin{array}{cc}A_{11} & A_{12} \\ A_{21} & A_{22}\end{array}\right]=\tilde{T} \tilde{A} \tilde{T}^{-1}$ with $A_{22} \in R^{m \times m}, B=\left[\begin{array}{c}0 \\ B_{2}\end{array}\right]=\tilde{T} \tilde{B}$ with nonsingular matrix $B_{2} \in R^{m \times m}, C=\tilde{C} \tilde{T}^{-1}$ and $\Psi(t)=\tilde{T} \tilde{\Psi}(t)$.

The following Assumptions are imposed on system (2). 
Assumption 3. $\Psi(t)$ is a known continuous differentiable function and $\Psi(t)$ is bounded with $\|\Psi(t)\| \leqslant \rho_{1}$.

Assumption 4. $A_{12}$ is full row rank i.e. $\operatorname{rank}\left(A_{12}\right)=n-m$.

Remark 2. It should be pointed out that the disturbances considered in this paper are nonzero time-varying and independent of the system states. The derivative of the unmatched disturbances $d(t)$ considered in Yang et al., (2013) is required to satisfy $\lim _{t \rightarrow \infty} \dot{d}(t)=0$ while this condition is not required in this study.

Remark 3. It should be noted that Assumptions 1-3 are common and reasonable. Assumption 4 is not conservative and may be satisfied by many linear systems.

It follows from Assumption 1 that $(A, C)$ is also observable. Thus there exists a matrix $L \in R^{n \times p}$ such that $A-L C$ is stable. Therefore for any symmetric matrix $Q_{1}>0$, the Lyapunov equation

$$
(A-L C)^{T} P_{1}+P_{1}(A-L C)=-Q_{1}
$$

has a unique symmetric solution $P_{1}>0$.

It follows from Proposition 3.3 in Edwards and Spurgeon (1998) that the pair $\left(A_{11}, A_{12}\right)$ is controllable. Thus there exists a matrix $K \in R^{m \times(n-m)}$ such that $A_{11}-$ $A_{12} K$ is stable. Then for any symmetric matrix $Q_{2}>0$, the Lyapunov equation

$$
\left(A_{11}-A_{12} K\right)^{T} P_{2}+P_{2}\left(A_{11}-A_{12} K\right)=-Q_{2}
$$

has a unique symmetric solution $P_{2}>0$.

The objective is to design a composite sliding surface formed by the system output, the observed state and the estimated parameters such that the reduced-order sliding mode is exponentially stable. For system (2), a dynamic output feedback control is defined of the following form

$$
u=u(t, \hat{x}, \hat{\theta}, y)
$$

where the joint state-parameter adaptive observers are described by

$$
\begin{gathered}
\dot{\hat{x}}=\hat{x}(t, u, \hat{x}, \hat{\theta}, y) \\
\dot{\hat{\theta}}=\hat{\theta}(t, u, \hat{x}, y)
\end{gathered}
$$

The system will be designed such that the associated closed-loop system formed by (2) and (5)-(7) can be driven to the pre-designed sliding surface in finite time and a sliding motion can be maintained thereafter.

\section{Sliding surface design and stability analysis of the sliding motion}

\section{Sliding surface design based on joint state-parameter adaptive observers}

The following state-parameter adaptive observers have been introduced as in Zhang (2002); Zhang and Clavel (2001)

$$
\dot{\hat{x}}=A \hat{x}+B u+\left(\Upsilon \Gamma^{-1} \Upsilon^{T} C^{T}+L\right)(y-C \hat{x})+\Psi(t) \hat{\theta}
$$




$$
\dot{\hat{\theta}}=\Gamma^{-1} \Upsilon^{T} C^{T}(y-C \hat{x})
$$

where $L$ satisfies (3). The parameter gain $\Gamma$ is designed by

$$
\dot{\Gamma}=-\rho_{2} \Gamma+\Upsilon^{T} C^{T} C \Upsilon
$$

where the forgetting factor $\rho_{2}$ is a sufficiently large positive constant, the initial value $\Gamma\left(t_{0}\right)$ is symmetric positive definite, and $\Upsilon$ is generated by the following dynamical system

$$
\dot{\Upsilon}=(A-L C) \Upsilon+\Psi(t)
$$

For (11), it is obvious that $\Upsilon$ is bounded since $(A-L C)$ is stable and $\Psi(t)$ is bounded (Zhang (2002)). It follows that there exists a constant $\rho_{3}$ such that $\|\Upsilon\| \leqslant \rho_{3}$.

Define $e=x-\hat{x}, \tilde{\theta}=\theta-\hat{\theta}$ and notice that $\dot{\theta}=0$, then

$$
\begin{gathered}
\dot{e}=\left(A-L C-\Upsilon \Gamma^{-1} \Upsilon^{T} C^{T} C\right) e+\Psi(t) \tilde{\theta} \\
\dot{\tilde{\theta}}=-\Gamma^{-1} \Upsilon^{T} C^{T} C e
\end{gathered}
$$

Lemma 1. (Zhang and Clavel (2001)) If the forgetting factor $\rho_{2}>0$ and the initial gain matrix $\Gamma\left(t_{0}\right)$ is symmetric positive definite, then the gain matrix $\Gamma$ generated by (10) is positive definite and uniformly bounded, i.e. there exists a positive constant $\rho_{4}$ such that $\|\Gamma\| \leqslant \rho_{4}$. Moreover, if the state-parameter adaptive observers are designed as (8)-(9), then there exist two positive constants $\chi_{1}, \chi_{2}$ such that $\|e\| \leqslant \chi_{1},\|\tilde{\theta}\| \leqslant \chi_{2}$, where $\chi_{1}, \chi_{2}$ are determined by $\rho_{2}, L$ and the initial values of $e$ and $\tilde{\theta}$.

In order to facilitate the subsequent proof of convergence, the following definition linear combination of $e$ and $\tilde{\theta}$ is defined

$$
\eta=e-\Upsilon \tilde{\theta}
$$

where $\Upsilon$ has been defined in (11).

From (12)-(14), it follows that

$$
\dot{\eta}=(A-L C) \eta
$$

In the $(x, \tilde{\theta}, \eta)$ coordinate system, the following extended system can be described

$$
\left[\begin{array}{c}
\dot{x} \\
\dot{\tilde{\theta}} \\
\dot{\eta}
\end{array}\right]=\left[\begin{array}{ccc}
A & 0 & 0 \\
0 & -\Gamma^{-1} \Upsilon^{T} C^{T} C \Upsilon & -\Gamma^{-1} \Upsilon^{T} C^{T} C \\
0 & 0 & A-L C
\end{array}\right]\left[\begin{array}{c}
x \\
\tilde{\theta} \\
\eta
\end{array}\right]+\left[\begin{array}{c}
B \\
0 \\
0
\end{array}\right] u+\left[\begin{array}{c}
\Psi(t) \theta \\
0 \\
0
\end{array}\right]
$$

Remark 4. It should be noted that the information obtained from the observer design has not been used for control design in Zhang (2002); Zhang and Clavel (2001) and no strict Lyapunov proof has been provided. 
Remark 5. The extended system (16) contains all the necessary information on the variables and forms the basis for the subsequent design and stability analysis of the sliding mode control.

In order to design a stable sliding mode dynamics, a further transformation $z=T x$ will be given, where $T=\left[\begin{array}{cc}I_{n-m} & 0 \\ K & I_{m}\end{array}\right]$ and $K$ satisfies (4). In the new coordinates, system (2) can be described by:

$$
\dot{z}=\left[\begin{array}{cc}
\bar{A}_{11} & \bar{A}_{12} \\
\bar{A}_{21} & \bar{A}_{22}
\end{array}\right]\left[\begin{array}{c}
z_{1} \\
z_{2}
\end{array}\right]+\left[\begin{array}{c}
0 \\
B_{2}
\end{array}\right] u+\left[\begin{array}{c}
T_{1} \Psi(t) \theta \\
T_{2} \Psi(t) \theta
\end{array}\right]
$$

where $z:=\operatorname{col}\left(\begin{array}{cc}z_{1} & z_{2}\end{array}\right), z_{1} \in R^{n-m}, z_{2} \in R^{m}, \bar{A}_{11}=A_{11}-A_{12} K, \bar{A}_{12}=A_{12}$, $T_{1}:=\left[\begin{array}{ll}I_{n-m} & 0\end{array}\right] \in R^{(n-m) \times n}, T_{2}:=\left[\begin{array}{ll}K & I_{m}\end{array}\right] \in R^{m \times n}$.

For system (2), design the sliding function as

$$
\sigma(y, \hat{x})=S_{1} y+S_{2} \hat{x}+\bar{A}_{12}^{r i g h t} T_{1} \Psi(t) \hat{\theta}
$$

where $\bar{A}_{12}^{\text {right }}:=\bar{A}_{12}^{T}\left(\bar{A}_{12} \bar{A}_{12}^{T}\right)^{-1} \in R^{m \times(n-m)}$ is the right inverse of $\bar{A}_{12}, S_{1} \in R^{m \times p}$ is a design parameter, and $S_{2}=S-S_{1} C$ with $S=\left[\begin{array}{cc}0 & I_{m}\end{array}\right] T$.

Remark 6. Note that $\bar{A}_{11}$ is stable which can make the stability analysis of the sliding mode dynamics more straightforward than with the traditional dynamic OFSMC design, such as Yan et al., (2004, 2009b, 2010, 2006). Furthermore, an additional advantage is that an explicit expression can be given for $S, S_{1}, S_{2}$.

\section{Stability analysis of the sliding motion}

From (18), it follows that

$$
\begin{aligned}
\sigma(y, \hat{x}, \hat{\theta}) & =S_{1} y+S_{2} \hat{x}+\bar{A}_{12}^{\text {right }} T_{1} \Psi(t) \hat{\theta} \\
& =S_{1} C x+S_{2}(x-e)+\bar{A}_{12}^{r i g h t} T_{1} \Psi(t) \hat{\theta} \\
& =S x-S_{2} e+\bar{A}_{12}^{\text {right }} T_{1} \Psi(t) \hat{\theta} \\
& =S T^{-1} z-S_{2} e+\bar{A}_{12}^{\text {right }} T_{1} \Psi(t) \hat{\theta} \\
& =z_{2}-S_{2} e+\bar{A}_{12}^{\text {right }} T_{1} \Psi(t) \hat{\theta}
\end{aligned}
$$

In the new coordinates, the sliding surface is given by:

$$
z_{2}=S_{2} e-\bar{A}_{12}^{r i g h t} T_{1} \Psi(t) \hat{\theta}
$$

When system (16) is restricted to the sliding surface (20), the corresponding extended system dynamics are described by

$$
\left[\begin{array}{c}
\dot{z}_{1} \\
\dot{\tilde{\theta}} \\
\dot{\eta}
\end{array}\right]=\left[\begin{array}{ccc}
\bar{A}_{11} & \bar{A}_{12} S_{2} \Upsilon+T_{1} \Psi(t) & \bar{A}_{12} S_{2} \\
0 & -\Gamma^{-1} \Upsilon^{T} C^{T} C \Upsilon & -\Gamma^{-1} \Upsilon^{T} C^{T} C \\
0 & 0 & A-L C
\end{array}\right]\left[\begin{array}{c}
z_{1} \\
\tilde{\theta} \\
\eta
\end{array}\right]
$$


Theorem 1. Suppose Assumptions 1-4 are satisfied. Then, the extended system (21) is exponentially stable if $M>0$ with $M$ defined by

$M=\left[\begin{array}{ccc}\underline{\lambda}\left(Q_{2}\right) & -\left\|P_{2} \bar{A}_{12} S_{2}\right\| & -\rho_{3}\left\|P_{2} \bar{A}_{12} S_{2}\right\|-\rho_{1}\left\|P_{2} T_{1}\right\| \\ -\left\|P_{2} \bar{A}_{12} S_{2}\right\| & \underline{\lambda}\left(Q_{1}\right)-\bar{\lambda}\left(C^{T} C\right) & 0 \\ -\rho_{3}\left\|P_{2} \bar{A}_{12} S_{2}\right\|-\rho_{1}\left\|P_{2} T_{1}\right\| & 0 & \rho_{2} \rho_{4}\end{array}\right]$

\section{Proof:}

For system (21), consider the Lyapunov function candidate

$$
V\left(z_{1}, \eta, \tilde{\theta}, t\right)=z_{1}^{T} P_{2} z_{1}+\eta^{T} P_{1} \eta+\tilde{\theta}^{T} \Gamma \tilde{\theta}
$$

The time derivative of $V$ along the trajectories of system (21) is given as

$$
\begin{aligned}
\dot{V} & =-z_{1}^{T} Q_{2} z_{1}+2 z_{1}^{T} P_{2} \bar{A}_{12} S_{2}(\eta+\Upsilon \tilde{\theta})+2 z_{1}^{T} P_{2} T_{1} \Psi(t) \tilde{\theta} \\
& -\eta^{T} Q_{1} \eta-2 \tilde{\theta}^{T} \Gamma \Gamma^{-1} \Upsilon^{T} C^{T} C(\eta+\Upsilon \tilde{\theta})+\tilde{\theta}^{T}\left(-\rho_{2} \Gamma+\Upsilon^{T} C^{T} C \Upsilon\right) \tilde{\theta} \\
& =-z_{1}^{T} Q_{2} z_{1}+2 z_{1}^{T} P_{2} \bar{A}_{12} S_{2}(\eta+\Upsilon \tilde{\theta})+2 z_{1}^{T} P_{2} T_{1} \Psi(t) \tilde{\theta} \\
& -\eta^{T} Q_{1} \eta-\rho_{2} \tilde{\theta}^{T} \Gamma \tilde{\theta}-2 \tilde{\theta}^{T} \Upsilon^{T} C^{T} C \eta-\tilde{\theta}^{T} \Upsilon^{T} C^{T} C \Upsilon \tilde{\theta}
\end{aligned}
$$

Noted that there exists the following relationship

$$
\begin{aligned}
-2 \tilde{\theta}^{T} \Upsilon^{T} C^{T} C \eta-\tilde{\theta}^{T} \Upsilon^{T} C^{T} C \Upsilon \tilde{\theta} & =-(\eta+\Upsilon \tilde{\theta})^{T} C^{T} C(\eta+\Upsilon \tilde{\theta})+\eta^{T} C^{T} C \eta \\
& \leqslant \eta^{T} C^{T} C \eta
\end{aligned}
$$

Then (24) can be further described by

$$
\begin{aligned}
\dot{V} & \leqslant-z_{1}^{T} Q_{2} z_{1}+2 z_{1}^{T} P_{2} \bar{A}_{12} S_{2}(\eta+\Upsilon \tilde{\theta})+2 z_{1}^{T} P_{2} T_{1} \Psi(t) \tilde{\theta}-\eta^{T} Q_{1} \eta-\rho_{2} \tilde{\theta}^{T} \Gamma \tilde{\theta}+\eta^{T} C^{T} C \eta \\
& \leqslant-\underline{\lambda}\left(Q_{2}\right)\left\|z_{1}\right\|^{2}+2\left\|P_{2} \bar{A}_{12} S_{2}\right\|\left\|z_{1}\right\|\|\eta\|+2\left(\rho_{3}\left\|P_{2} \bar{A}_{12} S_{2}\right\|+\rho_{1}\left\|P_{2} T_{1}\right\|\right)\left\|z_{1}\right\|\|\tilde{\theta}\| \\
& +\left\{\bar{\lambda}\left(C^{T} C\right)-\underline{\lambda}\left(Q_{1}\right)\right\}\|\eta\|^{2}-\rho_{2} \rho_{4}\|\tilde{\theta}\|^{2} \\
& =-X^{T} M X
\end{aligned}
$$

where $X=\left[\begin{array}{lll}\left\|z_{1}\right\| & \|\eta\| & \| \tilde{\theta}\end{array}\right]^{T}$.

It follows that

$$
\dot{V} \leqslant-\underline{\lambda}(M)\|X\|^{2}
$$


It should be pointed that although the gain matrix $\Gamma$ is positive define, it may be not symmetric. Hence,

$$
\begin{aligned}
\tilde{\theta}^{T} \Gamma \tilde{\theta} & =\tilde{\theta}^{T}\left(\frac{\Gamma+\Gamma^{T}}{2}+\frac{\Gamma-\Gamma^{T}}{2}\right) \tilde{\theta} \\
& =\tilde{\theta}^{T}\left(\frac{\Gamma+\Gamma^{T}}{2}\right) \tilde{\theta} \\
& \geqslant \underline{\lambda}\left(\frac{\Gamma+\Gamma^{T}}{2}\right)\|\tilde{\theta}\|^{2}
\end{aligned}
$$

It follows from (23) and (28) that

$$
k_{1}\left(\left\|z_{1}\right\|^{2}+\|\eta\|^{2}+\|\tilde{\theta}\|^{2}\right) \leqslant V\left(z_{1}, \eta, \tilde{\theta}, t\right) \leqslant k_{2}\left(\left\|z_{1}\right\|^{2}+\|\eta\|^{2}+\|\tilde{\theta}\|^{2}\right)
$$

where $k_{1}=\min \left\{\underline{\lambda}\left(P_{2}\right), \underline{\lambda}\left(P_{1}\right), \underline{\lambda}\left(\frac{\Gamma+\Gamma^{T}}{2}\right)\right\}, k_{2}=\max \left\{\bar{\lambda}\left(P_{2}\right), \bar{\lambda}\left(P_{1}\right), \rho_{4}\right\}$.

It follows from (27) and (29) that

$$
\dot{V} \leqslant-\frac{\underline{\lambda}(M)}{k_{2}} V
$$

Then,

$$
V \leqslant V\left(t_{0}\right) \exp \left\{2 k_{3}\left(t-t_{0}\right)\right\}
$$

where $k_{3}=-\frac{\lambda(M)}{2 k_{2}}$ and $V\left(t_{0}\right)$ represents the initial value.

From (14), it is obvious that

$$
\|e\| \leqslant\|\eta\|+\rho_{3}\|\tilde{\theta}\|
$$

Furthermore, it follows from (29), (31) and (32) that

$$
\|e\| \leqslant\left(1+\rho_{3}\right) \sqrt{\frac{V\left(t_{0}\right)}{k_{1}}} \exp \left\{k_{3}\left(t-t_{0}\right)\right\}
$$

Hence, the extended system (21) is exponentially stable while the observation error and parameter estimation error converge to zero exponentially.

\section{Sliding mode control design}

Based on the observed state, the estimated parameters given by (8)-(9) and the system output, the following sliding mode control is proposed

$$
\begin{aligned}
u & =-(S B)^{-1}\left\{S A \hat{x}+\left(S_{2} L+S_{2} \Upsilon \Gamma^{-1} \Upsilon^{T} C^{T}+\bar{A}_{12}^{r i g h t} T_{1} \Psi(t) \Gamma^{-1} \Upsilon^{T} C^{T}\right)(y-C \hat{x})\right. \\
& \left.+\left(\bar{A}_{12}^{r i g h t} T_{1} \dot{\Psi}(t)+S_{2} \Psi(t)\right) \hat{\theta}+\omega \frac{\sigma}{\|\sigma\|}\right\}
\end{aligned}
$$


where $\omega$ is defined by

$$
\omega=\mu+\chi_{1}\left\|S_{1} C A\right\|+\chi_{2}\left\|S_{1} C\right\|\|\Psi(t)\|+\left\|S_{1} C\right\|\|\Psi(t)\|\|\hat{\theta}\|
$$

with $\mu$ a positive constant.

Theorem 2. Suppose Assumptions 1-4 are satisfied. The control (34) with $\omega$ defined in (35) will drive the system (16) to the sliding surface in finite time and maintain a sliding motion thereafter.

\section{Proof:}

It follows from (16) and (18) that

$$
\begin{aligned}
\dot{\sigma} & =S B u+S A x+S \Psi(t) \theta-S_{2}\left(A-L C-\Upsilon \Gamma^{-1} \Upsilon^{T} C^{T} C\right) e \\
& -S_{2} \Psi(t) \tilde{\theta}+\bar{A}_{12}^{r i g h t} T_{1} \dot{\Psi}(t) \hat{\theta}+\bar{A}_{12}^{r i g h t} T_{1} \Psi(t) \Gamma^{-1} \Upsilon^{T} C^{T}(y-C \hat{x}) \\
& =S B u+S A x+S \Psi(t) \theta-S_{2} A e+S_{2} L C e+S_{2} \Upsilon \Gamma^{-1} \Upsilon^{T} C^{T} C e \\
& -S_{2} \Psi(t) \tilde{\theta}+\bar{A}_{12}^{r i g h t} T_{1} \dot{\Psi}(t) \hat{\theta}+\bar{A}_{12}^{r i g h t} T_{1} \Psi(t) \Gamma^{-1} \Upsilon^{T} C^{T}(y-C \hat{x}) \\
& =S B u+S A \hat{x}+S_{1} C A e+S_{1} C \Psi(t) \theta+S_{2} \Psi(t) \hat{\theta}+S_{2} L(y-C \hat{x}) \\
& +S_{2} \Upsilon \Gamma^{-1} \Upsilon^{T} C^{T}(y-C \hat{x})+\bar{A}_{12}^{r i g h t} T_{1} \dot{\Psi}(t) \hat{\theta}+\bar{A}_{12}^{r i g h t} T_{1} \Psi(t) \Gamma^{-1} \Upsilon^{T} C^{T}(y-C \hat{x})
\end{aligned}
$$

By applying the control (34) to (36),

$$
\begin{aligned}
\sigma^{T} \dot{\sigma} & =\sigma^{T}\left\{S B u+S A \hat{x}+S_{1} C A e+S_{1} C \Psi(t) \theta+S_{2} \Psi(t) \hat{\theta}+S_{2} L(y-C \hat{x})\right. \\
& \left.+S_{2} \Upsilon \Gamma^{-1} \Upsilon^{T} C^{T}(y-C \hat{x})+\bar{A}_{12}^{r i g h t} T_{1} \dot{\Psi}(t) \hat{\theta}+\bar{A}_{12}^{r i g h t} T_{1} \Psi(t) \Gamma^{-1} \Upsilon^{T} C^{T}(y-C \hat{x})\right\} \\
& =\sigma^{T}\left\{S_{1} C A e+S_{1} C \Psi(t) \theta-\omega \frac{\sigma}{\|\sigma\|}\right\} \\
& \leqslant-\|\sigma\|\left\{\omega-\left\|S_{1} C A\right\|\|e\|-\left\|S_{1} C\right\|\|\Psi(t)\|\|\theta\|\right\} \\
& \leqslant-\|\sigma\|\left\{\omega-\left\|S_{1} C A\right\|\|e\|-\left\|S_{1} C\right\|\|\Psi(t)\|(\|\tilde{\theta}\|+\|\hat{\theta}\|)\right\} \\
& \leqslant-\|\sigma\|\left\{\mu+\chi_{1}\left\|S_{1} C A\right\|+\chi_{2}\left\|S_{1} C\right\|\|\Psi(t)\|+\left\|S_{1} C\right\|\|\Psi(t)\|\|\hat{\theta}\|\right. \\
& \left.-\left\|S_{1} C A\right\|\|e\|-\|\tilde{\theta}\|\left\|S_{1} C\right\|\|\Psi(t)\|-\left\|S_{1} C\right\|\|\Psi(t)\|\|\hat{\theta}\|\right\} \\
& \leqslant-\mu\|\sigma\|
\end{aligned}
$$

According to the so-called $\eta$ reachability condition (Edwards and Spurgeon (1998)), Theorem 2 is proved.

Remark 7. According to the principles of state transformation, Theorems 1 and 2 are appropriate for the original system (1). One can use $\hat{\tilde{x}}=\tilde{T}^{-1} \hat{x}$ and $\tilde{x}=\tilde{T}^{-1} x$ directly in the controller implementation. 


\section{Simulation example}

The proposed approach will now be validated by a practically oriented example. Consider the lateral dynamics of the HIRM aircraft at the trim values of Mach 0.2 and height 5000 $\mathrm{ft}$ taken from Kureemun (2002). The system matrices are given by (see Yan et al., (2004))

$$
\begin{aligned}
& \tilde{A}=\left[\begin{array}{cccc}
-0.0080 & 0.4100 & -0.9047 & 0.1334 \\
-7.3235 & -0.4278 & 2.6462 & 0 \\
-0.1460 & -0.0247 & -0.1544 & 0 \\
0 & 1.0000 & 0.4558 & 0
\end{array}\right], \tilde{B}=\left[\begin{array}{cc}
0.0181 & -0.0094 \\
-3.1026 & 0.4024 \\
-0.4096 & -0.0833 \\
0 & 0
\end{array}\right] \\
& \tilde{C}=\left[\begin{array}{llll}
1 & 0 & 0 & 0 \\
0 & 0 & 1 & 0
\end{array}\right]
\end{aligned}
$$

For the lateral dynamics of the HIRM aircraft, the system states $\tilde{x}:=\operatorname{col}\left(\tilde{x}_{1}, \tilde{x}_{2}, \tilde{x}_{3}, \tilde{x}_{4}\right)$ represent sideslip angle $(\mathrm{rad})$, roll rate $(\mathrm{rad} / \mathrm{s})$, yaw rate $(\mathrm{rad} / \mathrm{s})$ and bank angle (rad) respectively, the system inputs $u:=\operatorname{col}\left(u_{1}, u_{2}\right)$ represent differential tailplane deflection (rad) and differential canard deflection (rad) respectively, the system outputs $y:=\operatorname{col}\left(y_{1}, y_{2}\right)$ represent sideslip angle (rad) and yaw rate (rad/s) respectively. The system zeros are 32.3877 and -0.3254 and thus it is a non-minimum phase system. Suppose that the system suffers from the disturbances $\tilde{\Psi}(t) \theta$ where $\tilde{\Psi}(t)$ is given by

$$
\tilde{\Psi}(t)=\left[\begin{array}{c}
0.0053 \sin t \\
-0.0130 \sin t \\
0.0990 \sin t \\
0.1 \cos t
\end{array}\right]^{T}
$$

and $\theta=0.3$.

The coordinate transformation $x=\tilde{T} \tilde{x}$ is given by

$$
\tilde{T}=\left[\begin{array}{cccc}
0.9986 & 0.0126 & -0.0516 & 0 \\
0 & 0 & 0 & 1 \\
-0.0058 & 0.9914 & 0.1309 & 0 \\
0.0528 & -0.1304 & 0.9901 & 0
\end{array}\right]
$$

Then in the new coordinates $x$, the system can be rewritten as

$$
\begin{aligned}
A & =\left[\begin{array}{cccc}
-0.0433 & 0.1332 & 0.2895 & -0.9112 \\
-0.0109 & 0 & 1.0510 & 0.3209 \\
-7.4092 & -0.0008 & -0.0425 & 2.2539 \\
0.8377 & 0.0070 & -0.0236 & -0.5044
\end{array}\right], B=\left[\begin{array}{cc}
0 & 0 \\
0 & 0 \\
-3.1296 & 0.3881 \\
0 & -0.1354
\end{array}\right] \\
C & =\left[\begin{array}{cccc}
0.9986 & 0 & -0.0058 & 0.0528 \\
-0.0516 & 0 & 0.1309 & 0.9901
\end{array}\right]
\end{aligned}
$$

and $\Psi(t)=0.1\left[\begin{array}{llll}0 & \cos t & 0 & \sin t\end{array}\right]^{T}$. 
Since $(A, C)$ is observable, design

$$
L=\left[\begin{array}{cc}
1.4049 & 0.7594 \\
3.7481 & 19.2627 \\
-7.2881 & 1.0807 \\
0.8892 & 0.8237
\end{array}\right]
$$

such that the eigenvalues of $A-L C$ are $\left[\begin{array}{llll}-1 & -1 & -0.5 & -0.5\end{array}\right]^{T}$. Then choosing $Q_{1}=2 I_{4}$, the solution of the Lyapunov equation (3) is

$$
P_{1}=\left[\begin{array}{cccc}
0.1084 & -0.0550 & 0.0523 & 0.6934 \\
-0.0550 & 0.0369 & -0.0286 & -0.3821 \\
0.0523 & -0.0286 & 0.0570 & 0.3878 \\
0.6934 & -0.3821 & 0.3878 & 4.8671
\end{array}\right]
$$

Since $\left(A_{11}, A_{12}\right)$ is controllable, design

$$
K=\left[\begin{array}{cc}
1.1990 & 1.7753 \\
-3.9614 & 0.4179
\end{array}\right]
$$

Choosing $Q_{2}=I_{2}$, the solution of the Lyapunov equation (4) is

$$
P_{2}=\left[\begin{array}{cc}
0.1250 & 0 \\
0 & 0.2500
\end{array}\right]
$$

The further transformation $z=T x$ is given by

$$
T=\left[\begin{array}{cccc}
1 & 0 & 0 & 0 \\
0 & 1 & 0 & 0 \\
1.1990 & 1.7753 & 1 & 0 \\
-3.9614 & 0.4179 & 0 & 1
\end{array}\right]
$$

The main parameters of the sliding function (18) are given as:

$$
\begin{aligned}
& S=\left[\begin{array}{cccc}
1.1990 & 1.7753 & 1.00 & 0 \\
-3.9614 & 0.4179 & 0 & 1.00
\end{array}\right], S_{1}=\left[\begin{array}{cc}
0.1 & 0.2 \\
-0.1 & 0.2
\end{array}\right] \\
& \bar{A}_{12}^{\text {right }}=\left[\begin{array}{cc}
0.3054 & 0.8673 \\
-1.0004 & 0.2756
\end{array}\right]
\end{aligned}
$$

Based on the above analysis, Assumptions 1-4 and Lemma 1 are all satisfied with $\rho_{1}=0.1, \rho_{2}=5$.

Meanwhile, by direct computation, it follows that $\rho_{3}=2, \rho_{4}=2$ and

$$
M=\left[\begin{array}{ccc}
1 & -0.5620 & -1.1491 \\
-0.5620 & 1 & 0 \\
-1.1491 & 0 & 10
\end{array}\right]
$$


Thus the conditions of Theorem 1 are satisfied. The parameters of the control (34) are given as:

$$
\mu=0.5, \chi_{1}=0.6, \chi_{2}=0.4
$$

which guarantee that Theorem 2 holds.

For simulation purposes, $\sigma /(\|\sigma\|+\beta)$ is used to replace $\sigma /\|\sigma\|$ to reduce the chattering with $\beta=0.01$.

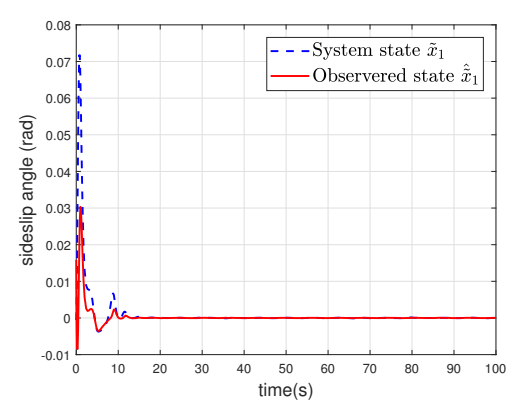

(a) The response of the sideslip angle and its estimate

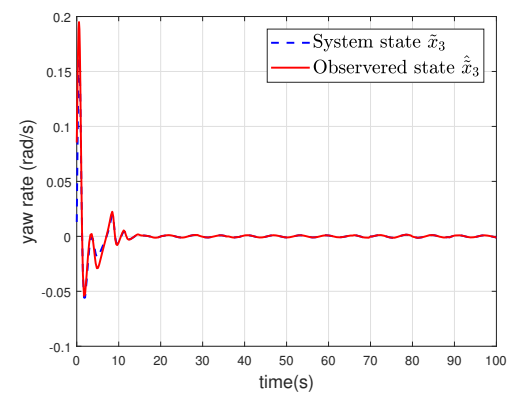

(c) The response of the yaw rate and its estimate

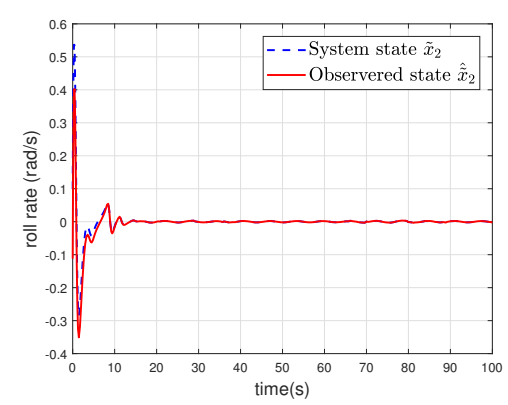

(b) The response of the roll rate and its estimate

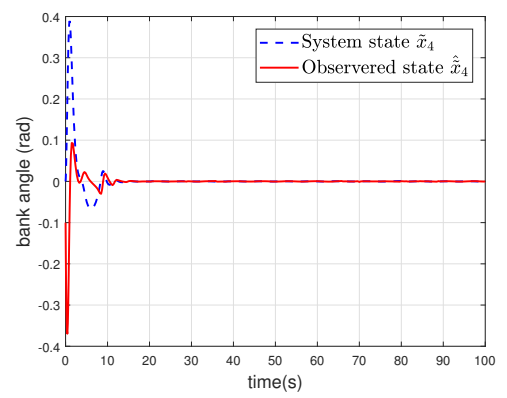

(d) The response of bank angle and its estimate

Figure 1. The time responses of the system states and their estimates

The time responses of the system states and their estimates are shown in Fig.1. The time responses of the sliding functions $\sigma$ and the system control signal $u$ are shown in Fig.2. The parameter estimate is shown in Fig.3. From Figs.1-3, it can be seen that the proposed joint state-parameter adaptive observer can estimate the system states and the uncertain parameter effectively. The HIRM aircraft is stabilized despite the presence of unmatched disturbances with nonzero steady-state value. The simulation results show that the proposed disturbance observer-based dynamic OFSMC is effective.

To further test the proposed disturbance observer-based dynamic OFSMC, the results obtained in Yan et al., (2004) will be compared with the results in this paper. The 


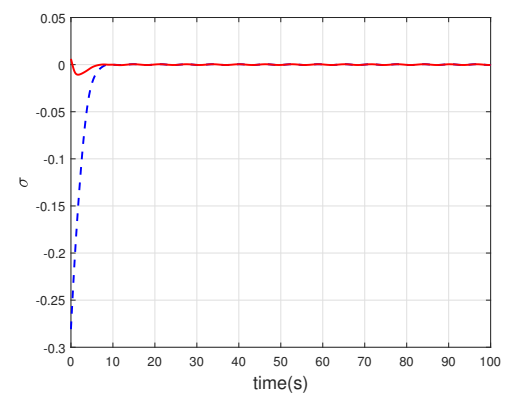

(a) The time response of the sliding function

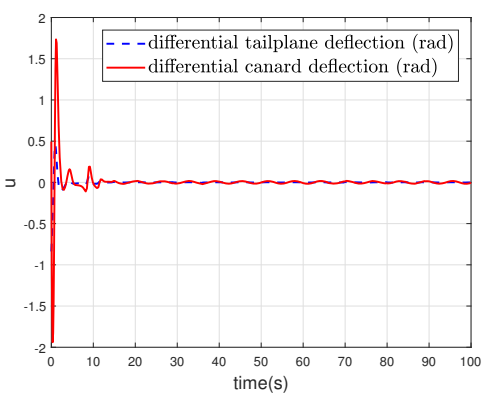

(b) The time response of the system control signal

Figure 2. The time responses of the sliding function and system control signal

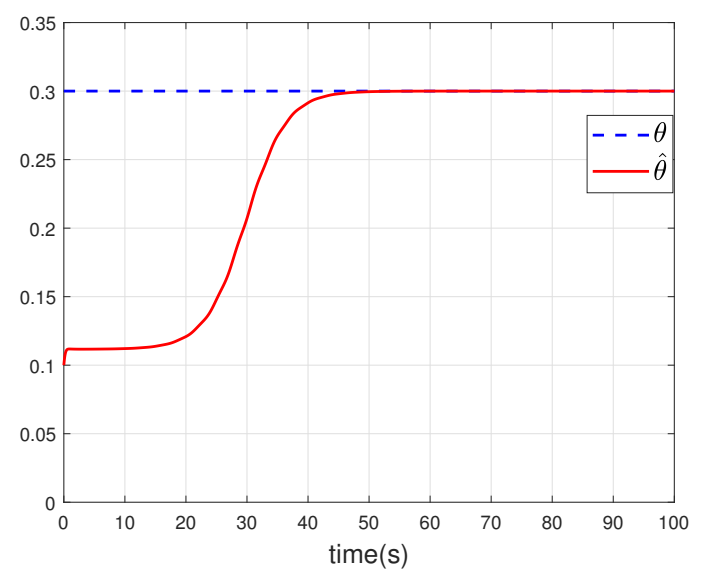

Figure 3. Estimate of the parameter and its actual value

selection of the control parameters is the same as those in Yan et al., (2004). The time responses of the system states and their estimates using the dynamic OFSMC proposed in Yan et al., (2004) are shown in Fig.4. The time responses of the sliding functions $\sigma$ and the system control signal $u$ are shown in Fig.5. Compared with Figs.1-5, the proposed dynamic OFSMC has a faster response speed and better system performance due to its direct consideration of the unmatched disturbances with nonzero steady-state value.

It should be noted that $\mu, \chi_{1}$ and $\chi_{2}$ are important factors to ensure that the considerec system can reach the sliding surface in finite time and the method of determining $\chi_{1}$ and $\chi_{2}$ has been given in Zhang (2002); Zhang and Clavel (2001). In addition, the state observer gain $L$ in (3) and the forgetting factor $\rho_{2}$ in (10) determine the convergence rate of the state observation error and parameter estimation error respectively. The following 


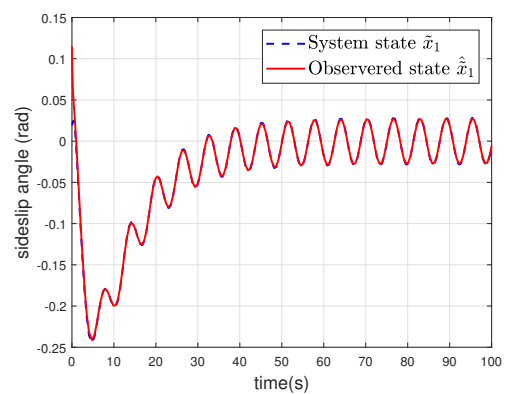

(a) The response of the sideslip angle and its estimate

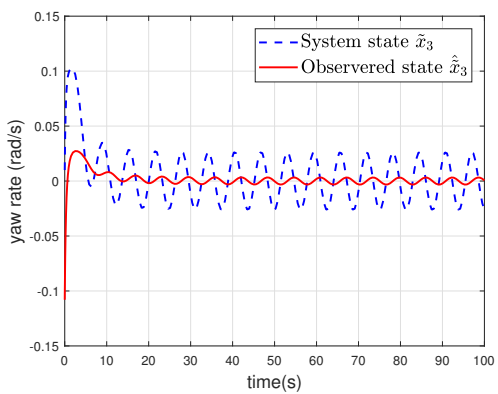

(c) The response of the yaw rate and its estimate

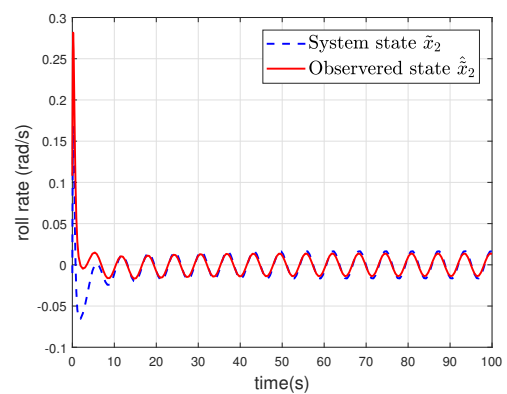

(b) The response of the roll rate and its estimate

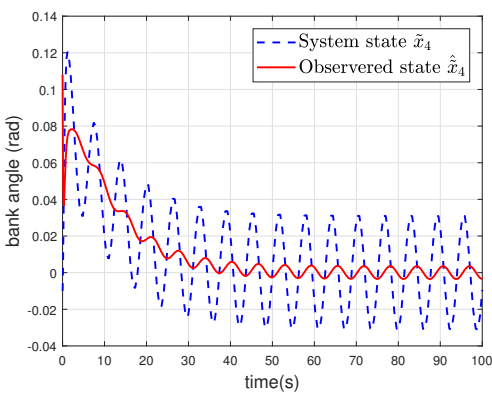

(d) The response of bank angle and its estimate

Figure 4. The time responses of the system states and their estimates using the dynamics OFSMC proposed in Yan et al., (2004)

simulation results will further show the influence of $\mu$ on the time for the system to reach
the sliding surface and the influence of $L$ and $\rho_{2}$ on system performance. Without loss
of generality, only the time response of the system state $\tilde{x}_{1}$, the sliding function $\sigma$, the
system control signal $u$ and the estimate of the parameter are given. All simulation times
are selected as 100 s.
i. Keeping the other parameters in (42)-(49) unchanged, the values of $\mu$ are chosen as
$0.005,0.05,0.5,5,50$ respectively. The time for the system to reach the sliding surface
and the maximum of the norm of $u$ are shown in Table 1 . The time response of the system
under the condition $\mu=50$ is shown in Fig.6. The time response of the system under the
condition $\mu=0.005$ is shown in Fig.7. By comparing Figs.1-3 with Figs.6-7 and Table 1 ,
with the increase of $\mu$, the time for the system to reach the sliding surface will be shorter
while the system input will gradually increase. In applications, the trial and error method
can be used to find an appropriate $\mu$.
ii. Keeping the other parameters in (42)-(49) unchanged, the value of $L$ only
is changed. Choose $L=L_{1}, L_{2}, L_{3}, L_{4}, L_{5}$ by pole placement so that the




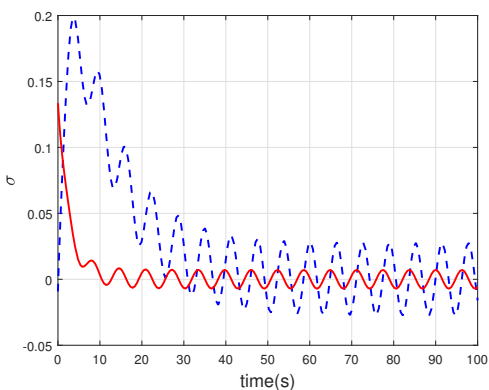

(a) The time response of the sliding function

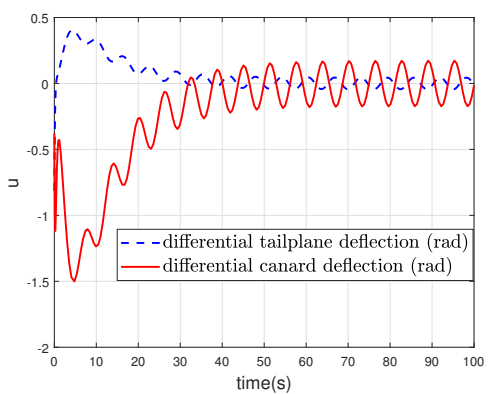

(b) The time response of the system control signal

Figure 5. The time responses of the sliding function and system control signal using the dynamics OFSMC proposed in Yan et al., (2004).

Table 1. The test for $\mu$

\begin{tabular}{ccc}
\hline$\mu$ & The time for the system to reach the sliding surface (s) & $\|u\|_{\max }$ \\
\hline 0.005 & 14.3 & 1.52 \\
0.05 & 13.1 & 1.77 \\
0.5 & 10.2 & 1.95 \\
5 & 2.5 & 2.86 \\
50 & 0.2 & 15.08 \\
\hline
\end{tabular}

Table 2. The test for $L$

\begin{tabular}{ccccc}
\hline $\mathrm{L}$ & $J_{1}$ & $J_{2}$ & $J_{3}$ & $\|u\|_{\max }$ \\
\hline$L_{1}$ & 7397.1260 & 5.5873 & 6.9203 & 2 \\
$L_{2}$ & 5875.9461 & 4.3763 & 4.8650 & 1.98 \\
$L_{3}$ & 17.5799 & 1.3640 & 2.4049 & 1.95 \\
$L_{4}$ & 41.2148 & 7.6724 & 3.1824 & 568 \\
$L_{5}$ & 1173.1036 & 16.7710 & 6.0929 & 1438 \\
\hline
\end{tabular}

\footnotetext{
eigenvalues of $A-L C$ are $0.01 \Omega, 0.1 \Omega, \Omega, 10 \Omega, 100 \Omega$ respectively, with $\Omega=$ $\left[\begin{array}{llll}-1 & -1 & -0.5 & -0.5\end{array}\right]^{T}$. The integral time multiplied square error (ITSE) indexes $J_{1}=\int_{0}^{\infty} t \tilde{\theta}^{2} d t, J_{2}=\int_{0}^{\infty} t e^{T} e d t$ and $J_{3}=\int_{0}^{\infty} t \tilde{x}^{T} \tilde{x} d t$ are introduced to analyze the system performance. The ITSE results and the maximum of the norm of $u$ are showr in Table 2. The time response of the system under the condition $L=L_{1}$ is shown in Fig.8. The time response of the system under the condition $L=L_{5}$ is shown in Fig.9 By comparing Figs.1-3 with Figs.8-9 and Table 2, with the decrease in the eigenvalues of $A-L C$, the convergence time of the state observation error reduces, but the system performance will first improve and then worsen, especially when the eigenvalue is very small, the system input will become very large.
} 


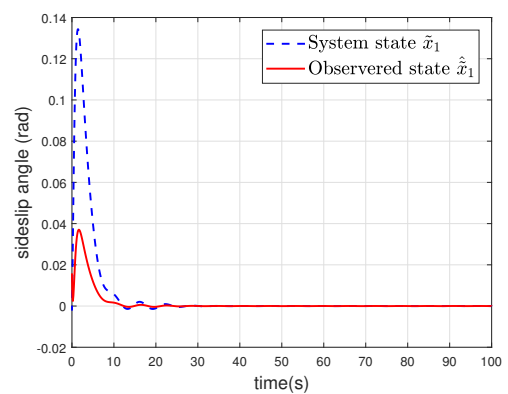

(a) The response of the sideslip angle and its estimate

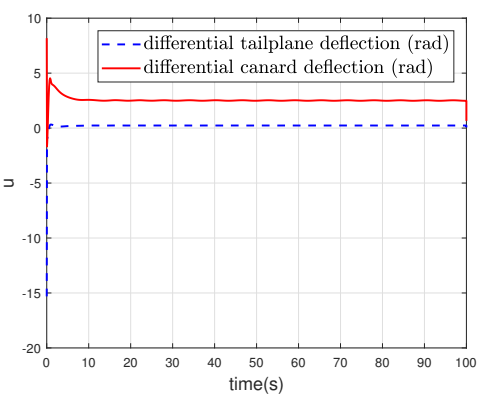

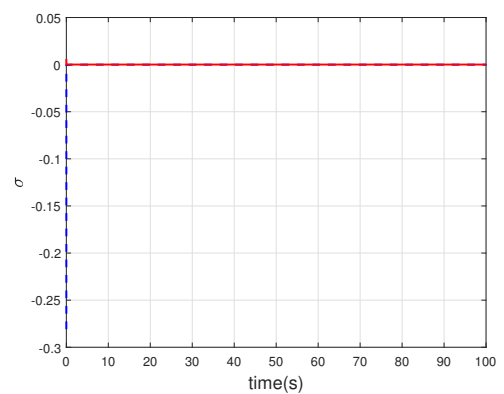

(b) The time response of the sliding function

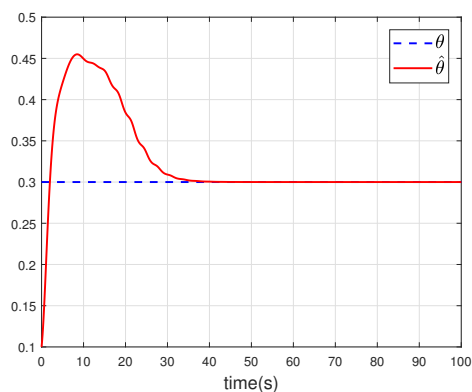

(c) The time response of the system control signal (d) Estimate of the parameter and its actual value

Figure 6. The time response of the system under the condition $\mu=50$

iii. Keeping the other parameters in (42)-(49) unchanged, the values of $\rho_{2}$ are chosen
as $0.05,0.5,5,50,500$ respectively. The ITSE results and the maximum of the norm of $u$
are shown in Table 3 . The time response of the system under the condition $\rho_{2}=0.05$ is
shown in Fig.10. The time response of the system under the condition $\rho_{2}=500$ is shown
in Fig.11. By comparing Figs.1-3 with Figs.10-11 and Table 3, with the increase of $\rho_{2}$,
the convergence time of the parameter estimation error will be shorter, but the system
performance will initially improve and then worsen, especially when $\rho_{2}$ is very large,
the system input will become very large. It should be noted that if $\rho_{2}$ is too small, the
parameter estimate will not converge to the actual value.

\section{Conclusion}

A dynamical OFSMC method has been proposed for a class of uncertain linear nonminimum phase systems. The unmatched disturbances can be allowed to have nonzero steady-state value. A sliding mode control has been designed to ensure that the system states reach the designed sliding surface in finite time. Simulation test results are given 


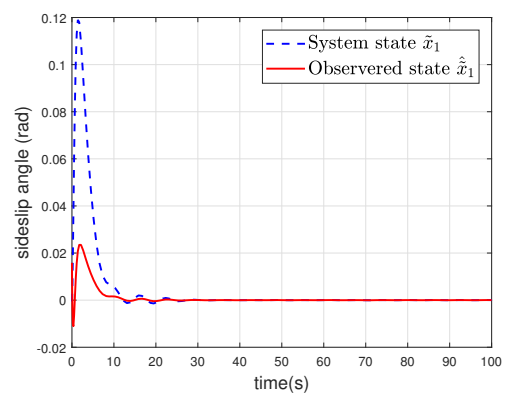

(a) The response of the sideslip angle and its estimate

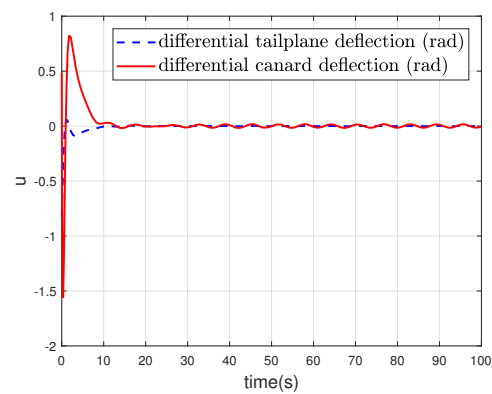

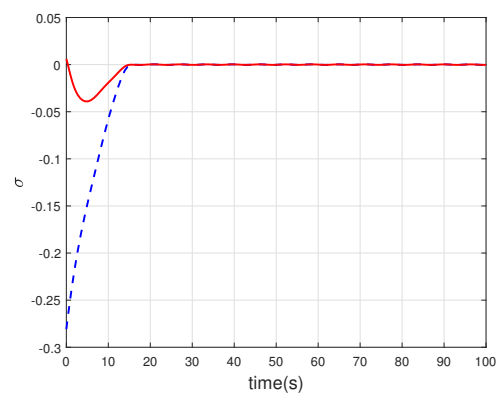

(b) The time response of the sliding function

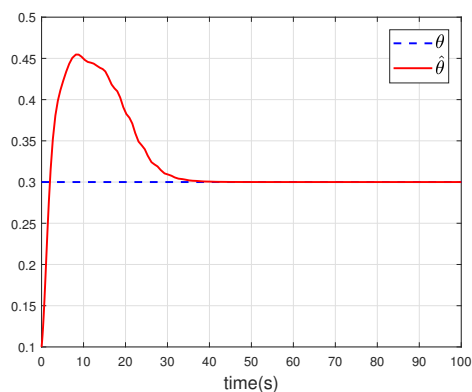

(c) The time response of the system control signal (d) Estimate of the parameter and its actual value

Figure 7. The time response of the system under the condition $\mu=0.005$

Table 3. The test for $\rho_{2}$

\begin{tabular}{ccccc}
\hline$\rho_{2}$ & $J_{1}$ & $J_{2}$ & $J_{3}$ & $\|u\|_{\max }$ \\
\hline 0.05 & 26.7828 & 9.5900 & 11.8311 & 1.6082 \\
0.5 & 20.3254 & 8.5410 & 11.2451 & 1.7392 \\
5 & 17.5799 & 1.3640 & 2.4049 & 1.95 \\
50 & 110.6306 & 15.5977 & 20.2541 & 14.3216 \\
500 & 3463.8769 & 21.1690 & 27.5700 & 136.2214 \\
\hline
\end{tabular}

to show the effectiveness of the proposed control scheme. Future work will focus on the application of the proposed method to interconnected systems.

\section{Acknowledgements}

This work is partially supported by the National Nature Science Foundation of China under grant nos 61973315, 61473312 . 


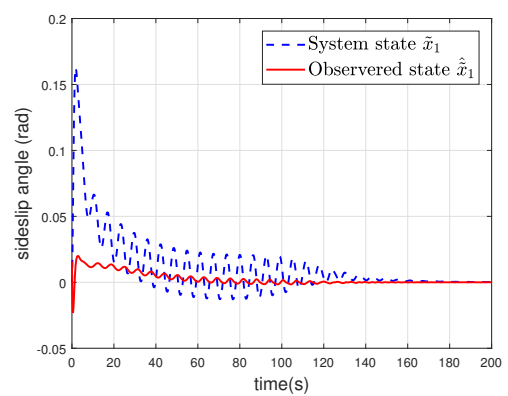

(a) The response of the sideslip angle and its estimate

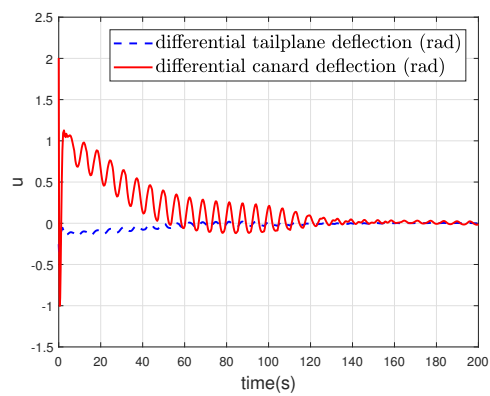

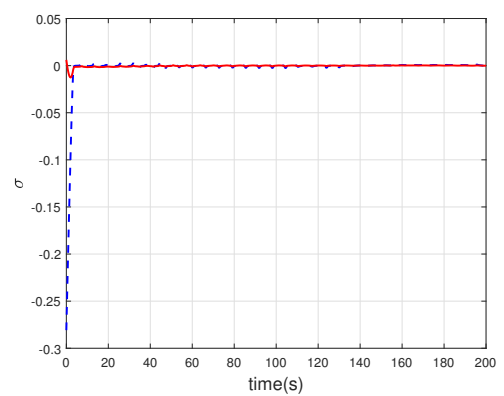

(b) The time response of the sliding function

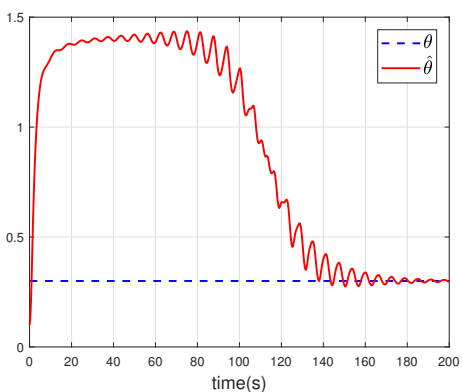

(c) The time response of the system control signal (d) Estimate of the parameter and its actual value

Figure 8. The time response of the system under the condition $L=L_{1}$

\section{References}

Benosman M and Vey G.-L (2003) Stable inversion of siso nonminimum phase linear systems through output planning: an experimental application to the one-link flexible manipulator. IEEE Transactions on Control Systems Technology 11(4): 588-597.

Cao W.-J and Xu J.-X (2004) Nonlinear integral-type sliding surface for both matched and unmatched uncertain systems. IEEE Transactions on Automatic Control 49(8): 1355-1360.

Chen W.-H, Ballance D.-J, et al. (2000) A nonlinear disturbance observer for robotic manipulators. IEEE Transactions on industrial Electronics 47(4): 932-938.

Chen W.-H (2003) Nonlinear disturbance observer-enhanced dynamic inversion control of missiles. Journal of Guidance, Control, and Dynamics 26(1): 161-166.

Consolini L and Tosques M (2012) A minimum phase output in the exact tracking problem for the nonminimum phase underactuated surface ship. IEEE Transactions on Automatic Control 57(12): 3174-3180.

Ding S and Li S (2017) Second-order sliding mode controller design subject to mismatched term. Automatica 77(3): 388-392. 


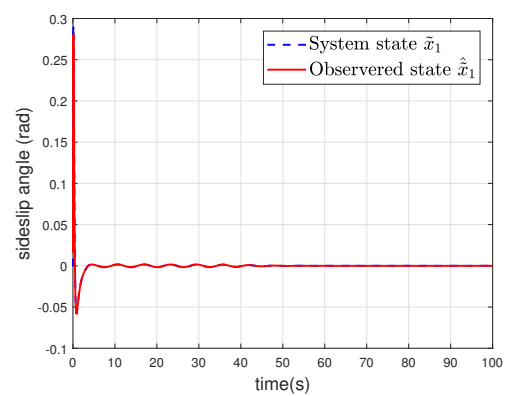

(a) The response of the sideslip angle and its estimate

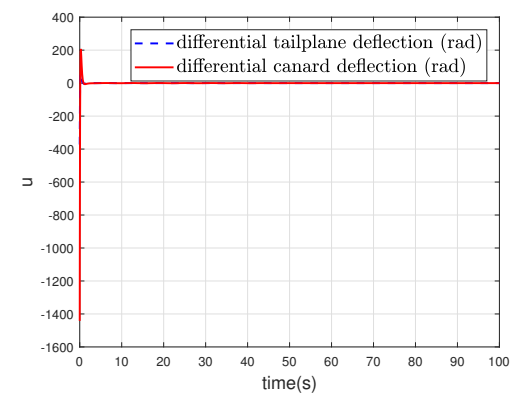

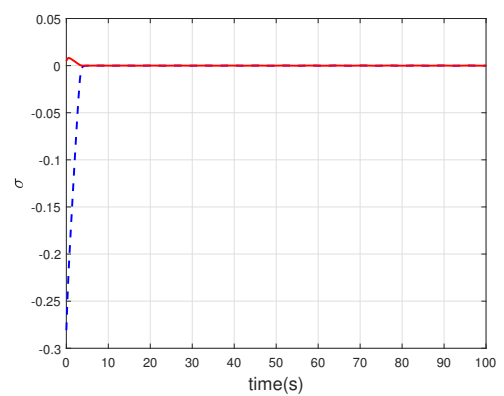

(b) The time response of the sliding function

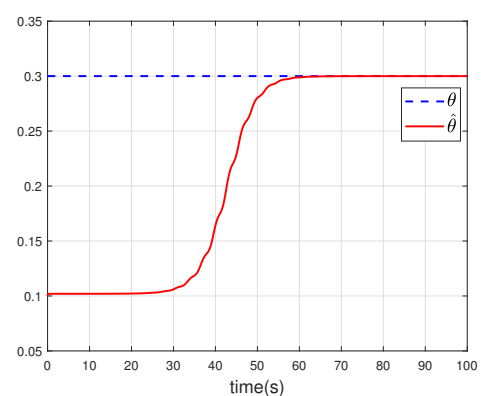

(c) The time response of the system control signal (d) Estimate of the parameter and its actual value

Figure 9. The time response of the system under the condition $L=L_{5}$.

Edwards C and Spurgeon S (1995) Sliding mode stabilization of uncertain systems using only output information. International Journal of Control 62(5): 1129-1144.

Edwards C and Spurgeon S (1998) Sliding mode control: theory and applications. London, UK: Taylor and Francis.

Edwards C, Yan X.-G and Spurgeon S (2007) On the solvability of the constrained lyapunov problem. IEEE Transactions on Automatic Control 52(10): 1982-1987.

Elkhazali R and Decarlo R (1995) Output feedback variable structure control design. Automatica 31(6): 805-816.

Galimidi A and Barmish B (1986) The constrained lyapunov problem and its application to robust output feedback stabilization. IEEE Transactions on Automatic Control 31(5): 410-419.

Gao R, Zhai D and Cheng J (2019) Decentralized static output feedback sliding mode control for interconnected descriptor systems via linear sliding variable. Applied Mathematics and Computation 357(9): 185-198.

Ginoya D, Shendge P and Phadke S (2013) Sliding mode control for mismatched uncertain systems using an extended disturbance observer. IEEE Transactions on Industrial Electronics 61(4): 


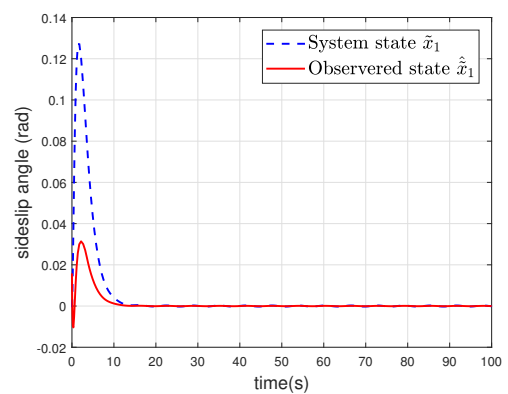

(a) The response of the sideslip angle and its estimate

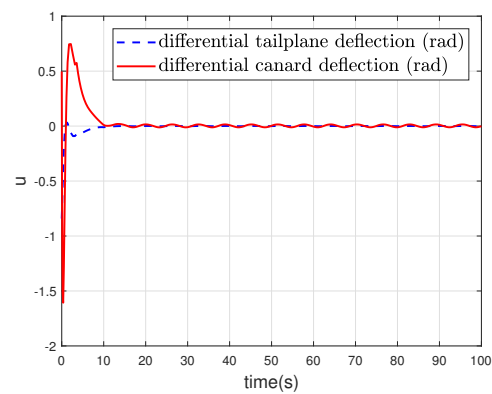

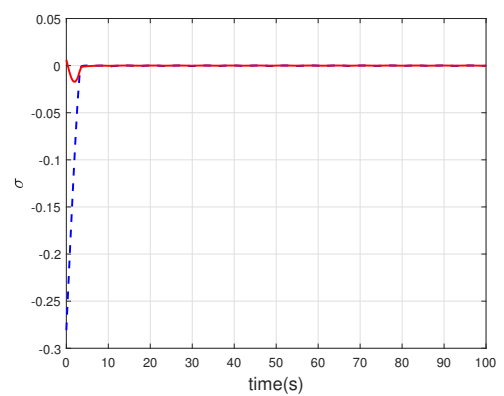

(b) The time response of the sliding function

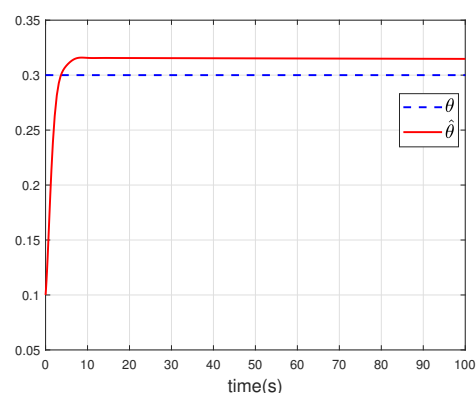

(c) The time response of the system control signal (d) Estimate of the parameter and its actual value

Figure 10. The time response of the system under the condition $\rho_{2}=0.05$

1983-1992.

Ji W, Qiu J and Karimi H.-R (2019) Fuzzy-model-based output feedback sliding-mode control for discrete-time uncertain nonlinear systems. IEEE Transactions on Fuzzy Systems 28(8): 15191530.

Jiang B.-P, Karimi H.R., Yang S.-C, Gao C.-C and Kao Y.-G (2021a) Observer-Based Adaptive Sliding Mode Control for Nonlinear Stochastic Markov Jump Systems via T-S Fuzzy Modeling: Applications to Robot Arm Model. IEEE Transactions on Industrial Electronics 68(1): 466-477.

Jiang B.-P, Gao C.-C (2021b) Decentralized adaptive sliding mode control of large-scale semi-Markovian jump interconnected systems with dead-zone input. IEEE Transactions on Automatic Control. Epub ahead of print 11 March 2021. DOI: 10.1109/TAC.2021.3065658.

Kim K.-S, Park Y and Oh S.-H (2000) Designing robust sliding hyperplanes for parametric uncertain systems: a riccati approach. Automatica 36(7): 1041-1048.

Kureemun, R., 2002, $\mu$-Analysis tools for the flight clearance of highly augmented aircraft, $\mathrm{PhD}$ Thesis: Department of Engineering, University of Leicester, UK. 


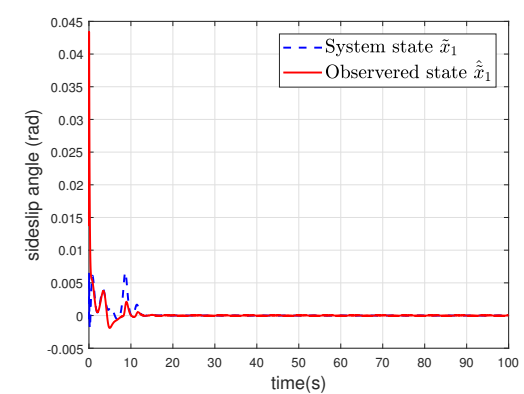

(a) The response of the sideslip angle and its estimate

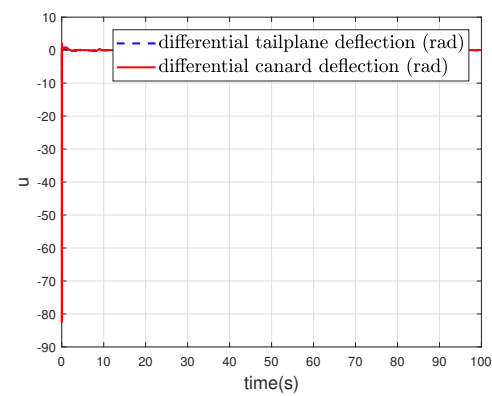

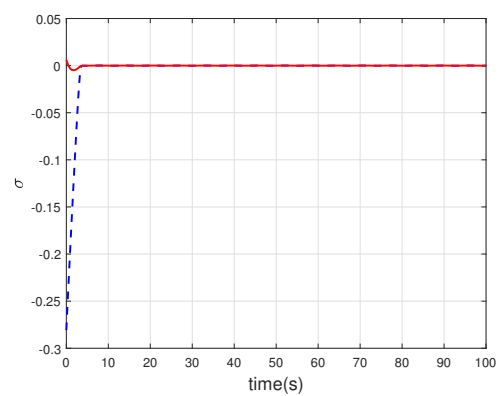

(b) The time response of the sliding function

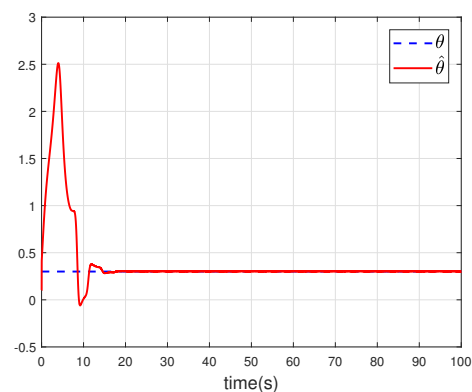

(c) The time response of the system control signal (d) Estimate of the parameter and its actual value

Figure 11. The time response of the system under the condition $\rho_{2}=500$

Mu J.-Q, Yan X.-G, et al. (2017) Decentralised sliding mode control for nonlinear interconnected systems in the generalised regular form. IFAC-PapersOnLine 50(1): 8850-8855.

Nguyen N.-P, Kim Y, et al. (2020) Disturbance observer-based continuous finite-time sliding mode control against matched and mismatched disturbances. Complexity 2020(5): 1-14.

Park P, Choi D.-J and Kong S.-G (2007) Output feedback variable structure control for linear systems with uncertainties and disturbances. Automatica 43(1): 72-79.

Plestan F, Shtessel Y, et al. (2010) New methodologies for adaptive sliding mode control. International Journal of Control 83(9): 1907-1919.

Utkin VI (1977) Variable structure systems with sliding modes. IEEE Transactions on Automatic control 22(2): 212-222.

Utkin VI (1992) Sliding modes in control and optimization. Springer, Berlin.

Wang J and Li S, et al. (2015) Extended state observer-based sliding mode control for pwm-based dc-dc buck power converter systems with mismatched disturbances. IET Control Theory and Applications 9(4): 579-586. 
Wen C.-C and Cheng C.-C (2008) Design of sliding surface for mismatched uncertain systems to achieve asymptotical stability. Journal of the Franklin Institute 345(8): 926-941.

Yang J, Li S.-H and Chen W.-H (2012) Nonlinear disturbance observer-based control for multiinput multi-output nonlinear systems subject to mismatching condition. International Journal of Control 85(8): 1071-1082.

Yang J, Li S.-H and Yu X.-H (2013) Sliding-mode control for systems with mismatched uncertainties via a disturbance observer. IEEE Transactions on Industrial Electronics 60(1): 160-169.

Yan X.-G, Edwards C and Spurgeon S (2004) Output feedback sliding mode control for nonminimum phase systems with non-linear disturbances. International Journal of control 77(15): 1353-1361.

Yan X.-G, Spurgeon S and Edwards C (2006) Decentralised sliding mode control for nonminimum phase interconnected systems based on a reduced-order compensator. Automatica 42(10): 1821-1828.

Yan X.-G, Spurgeon S and Edwards C (2009a) On discontinuous static output feedback control for linear systems with nonlinear disturbances. Systems and control letters 58(5): 314-319.

Yan X.-G, Spurgeon S and Edwards C (2009b) Global stabilisation for a class of nonlinear timedelay systems based on dynamical output feedback sliding mode control. International Journal of Control 82(12): 2293-2303.

Yan X.-G, Spurgeon S and Edwards C (2010) Sliding mode control for time-varying delayed systems based on a reduced-order observer. Automatica 46(8): 1354-1362.

Yan X.-G, Spurgeon S and Edwards C (2013) Decentralised stabilisation for nonlinear time delay interconnected systems using static output feedback. Automatica 49(2): 633-641.

Zhang Q.-H and Clavel A (2001) Adaptive observer with exponential forgetting factor for linear time varying systems. In: Proceedings of the 40th IEEE Conference on Decision and Control 4: 3886-3891.

Zhang Q.-H (2002) Adaptive observer for multiple-input-multiple-output (mimo) linear timevarying systems. IEEE Transactions on Automatic Control 47(3):525-529.

Zhang H, Wei X, et al. (2017) Disturbance rejection for nonlinear systems with mismatched disturbances based on disturbance observer. Journal of the Franklin Institute 354(11): 44044424.

Zhang Y and Yan P (2019) Adaptive observer-based integral sliding mode control of a piezoelectric nano-manipulator. IET Control Theory and Applications 13(14): 2173-2180. 